\title{
Gerontecnologias e internet das coisas para prevenção de quedas em idosos: revisão integrativa
}

Internet of things gerontechnology for fall prevention in older adults: an integrative review Gerontecnologías y internet de las cosas para prevención de caídas en adultos mayores: revisión integradora

Jamylle Lucas Diniz¹ id https://orcid.org/0000-0002-5697-8599

Viviane Ferreira Sousa ${ }^{1}$ id https://orcid.org/0000-0002-4456-0400

Janaína Fonseca Victor Coutinho' ${ }^{1}$ id https://orcid.org/0000-0001-7451-0132

Ítalo Linhares de Araújo ${ }^{1}$ iD https://orcid.org/0000-0002-9270-6654

Rossana Maria de Castro Andrade ${ }^{1}$ iD https://orcid.org/0000-0002-0186-2994

Joyce da Silva Costa ${ }^{1}$ id https://orcid.org/0000-0001-6481-3682

Rachel Gabriel Bastos Barbosa ${ }^{1}$ id https://orcid.org/0000-0003-4205-6910

Marilia Braga Marques ${ }^{1}$ id https://orcid.org/0000-0002-7483-1435 Como citar:
Diniz JL, Sousa VF, Coutinho JF, Araújo IL, Andrade RM, Costa JS, et al. Gerontecnologias e internet das coisas para prevenção de quedas em idosos: revisão integrativa. Acta Paul Enferm. 2022;35:eAPE003142.

DOI

http://dx.doi.org/10.37689/acta-ape/2022AR03142

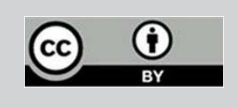

Descritores

Idoso; Internet das coisas; Tecnologia; Acidentes por quedas; Prevenção de acidentes

Keywords

Aged; Internet of things; Technology; Accidental falls; Accident prevention

Descriptores

Anciano; Internet de las cosas; Tecnología; Accidentes por caídas; Prevención de accidentes

Submetido 18 de Outubro de 2020

Aceito 26 de Maio de 2021

\section{Autor correspondente}

Jamylle Lucas Diniz E-mail: jamylledz@hotmail.com

Editor Associado (Avaliação pelos pares): Ana Lucia de Moraes Horta (https://orcid.org/0000-0001-5643-3321) Escola Paulista de Enfermagem, Universidade Federal de São Paulo, São Paulo, SP, Brasil

\section{Resumo}

Objetivo: Identificar na literatura as gerontecnologias Internet das Coisas desenvolvidas para prevenção de acidentes por quedas em idosos.

Métodos: Revisão integrativa, realizada de janeiro a maio de 2020. Foram critérios de inclusão artigos, sem restrição de período ou idioma com indivíduos de 60 anos ou mais, que abordem a utilização de gerontecnologia Internet das Coisas para prevenção de quedas. Excluíram-se artigos duplicados. A busca foi realizada pela estratégia PIE (População, Intervenção, Efeito/Avaliação), resultando na pergunta: "Quais as gerontecnologias Internet das Coisas desenvolvidas para prevenção de acidentes por quedas em idosos disponíveis na literatura?". Foi realizada nas bases de dados MEDLINE/PubMed, LILACS, CINAHL, Scopus e Web of Science. Identificaram-se ano, tipo de estudo, país, profissionais envolvidos, desfecho, local de desenvolvimento e classificação em gerontecnologia Internet das Coisas e protótipos.

Resultados: Identificaram-se 23 gerontecnologias Internet das Coisas. Os anos de 2018 e 2019 apresentaram maiores números de publicações. Ocorreu predominância de estudos descritivos, por profissionais da ciência da computação e engenheiros e desenvolvidos na Europa, Ásia, América do Norte e Oceania. Encontraram-se oito gerontecnologias Internet das Coisas e 15 protótipos, sendo sete sensores, cinco dispositivos, três jogos sérios e sistemas, dois robôs e um exergames, realidade virtual e aplicativo. A maioria das gerontecnologias buscava melhora da mobilidade e equilíbrio, sendo cinco desenvolvidas em ambiente hospitalar e domiciliar, respectivamente.

Conclusão: As gerontecnologias Internet das Coisas podem ser utilizadas como recursos para auxiliar na prevenção de quedas e no fortalecimento da capacidade funcional. Todavia, fazem-se necessárias pesquisas futuras para analisar a eficácia deste tipo de tecnologia para prevenção de quedas em idosos.

\section{Abstract}

Objective: To identify in the literature the Internet of Things gerontechnology developed to prevent accidents by falls in older adults.

Methods: This integrative review was carried out from January to May 2020. Articles without period or language restriction with individuals aged 60 years or older addressing the use of Internet of Things gerontechnology for fall prevention were included. Duplicate articles were excluded. The search was performed by the PIE strategy (Population, Intervention, Effect/Assessment), resulting in the question: "What Internet of Things gerontechnology developed for accident prevention by falls in older adults available in the literature?". It was performed in the MEDLINE/PubMed, LILACS, CINAHL, Scopus and Web of Science databases. Year, type of study, country, professionals involved, outcome, development site and classification in Internet of Things gerontechnology and prototypes were identified. 
Results: Twenty-three Internet of Things gerontechnology were identified. The years 2018 and 2019 had higher numbers of publications. There was a predominance of descriptive studies by computer science professionals and engineers and developed in Europe, Asia, North America and Oceania. Eight Internet of Things gerontechnology and 15 prototypes were found, seven sensors, five devices, three serious games and systems, two robots and one exergames, virtual reality and application. Most gerontechnology sought to improve mobility and balance, five of which were developed in the hospital and home environments, respectively.

Conclusion: The Internet of Things gerontechnology can be used as resources to assist in fall prevention and strengthening functional capacity. However, future research is needed to analyze the effectiveness of this type of technology for fall prevention in older adults.

\section{Resumen}

Objetivo: Identificar en la literatura las gerontecnologías internet de las cosas desarrolladas para la prevención accidentes por caídas en adultos mayores.

Métodos: Revisión integradora, realizada de enero a mayo de 2020. Los criterios de inclusión fueron artículos, sin restricción de período 0 idioma, con individuos de 60 años o más, que abordaran la utilización de gerontecnologías internet de las cosas para la prevención de caídas. Se excluyeron artículos duplicados. La búsqueda fue realizada mediante la estrategia PIE (población, intervención, efecto/evaluación), que dio como resultado la pregunta “¿Cuáles son las gerontecnologías internet de las cosas desarrolladas para la prevención de accidentes por caídas en adultos mayores disponibles en la literatura?". Se realizó en las bases de datos MEDLINE/PubMed, LILACS, CINAHL, Scopus y Web of Science. Se identificó el año, tipo de estudio, país, profesionales involucrados, resultado, lugar de desarrollo y clasificación en gerontecnologías internet de las cosas.

Resultados: Se identificaron 23 gerontecnologías internet de las cosas. Los años 2018 y 2019 presentaron mayores números de publicaciones. Hubo predominancia de estudios descriptivos, por profesionales de ciencias de la comunicación e ingenieros y desarrollados en Europa, Asia, América del Norte y Oceanía. Se encontraron ocho gerontecnologías internet de las cosas y 15 prototipos, de los cuales siete eran sensores, cinco dispositivos, tres juegos serios y sistemas, dos robots y un exergames, realidad virtual y aplicación. La mayoría de los gerontecnologías buscaba una mejora de la movilidad y el equilibrio, de las cuales cinco fueron desarrolladas en ambiente hospitalario y domiciliario.

Conclusión: Las gerontecnologías internet de las cosas pueden ser utilizadas como recurso para ayudar en la prevención de caídas y en el fortalecimiento de la capacidad funcional. Sin embargo, es necesario llevar a cabo estudios futuros para analizar la eficacia de este tipo de tecnología para la prevención de caídas en adultos mayores.

\section{Introdução}

Com o envelhecimento da população mundial, a necessidade de suporte ao público idoso torna-se imperativa para apoiar as demandas inerentes ao processo de envelhecimento. Os dias atuais revelam profundas e constantes mudanças em que a tecnologia vem fazendo parte do cotidiano de todos.

Nesse sentido, o desenvolvimento de tecnologias voltadas especificamente para idosos tem seu maior expoente na Gerontecnologia, visto que esta procura fornecer respostas a duas principais tendências: o aumento da população idosa e o crescente avanço tecnológico. ${ }^{(1)} \mathrm{O}$ termo gerontecnologia surgiu nos anos 70 e foi idealizado por engenheiros, designers e gerontólogos. ${ }^{(2)}$ Conta com representação em âmbito internacional e nacional por meio da International Society for Gerontechnology e Sociedade Brasileira de Gerontecnologia, entidades que reúnem profissionais para o desenvolvimento de tecnologias voltadas à pessoa idosa.

A Gerontecnologia é o estudo da tecnologia associada ao envelhecimento para adequação dos recursos tecnológicos à saúde, moradia, mobilidade, comunicação, lazer, dentre outros, no intuito de manter nos idosos suas habilidades físicas e cognitivas, tornando-os mais autônomos e independentes, condiçôes imprescindíveis para garantir sua capacidade funcional. ${ }^{(3)}$

Como inovação tecnológica capaz de contribuir com a Gerontecnologia, tem-se a Internet das Coisas (IoT), uma vez que esta permite que objetos físicos vejam, ouçam, pensem, executem tarefas, compartilhem informaçôes, processem dados, capturem variáveis ambientais e mudanças externas por meio de uma rede sem fio, que se comunica usando a Internet, ${ }^{(4)}$ incorporando dispositivos, sensores, sistemas, aplicativos, dentre outros, para prover um monitoramento mais completo e visando a um maior cuidado para os idosos.

A IoT pode ser utilizada em diversas áreas, todavia a área da saúde é apontada com uma das mais beneficiadas, pois soluçôes IoT podem ser aplicadas com dispositvos vestíveis (wearables) e dispositivos presentes no domicílio (smart home) com o objetivo de auxiliar no cuidados de saúde, o que possibilita o surgimento do Ambient Assisted Living (AAL). ${ }^{(5)}$ Assim, a IoT pode revolucionar a prevençáo de problemas e agravos que podem ser evitados com o monitoramento em tempo real. 
Portanto, as gerontecnologias com solução IoT podem auxiliar na manutenção da saúde e na prevenção de agravos aos idosos. Destaca-se como agravo de maior relevância na população idosa os acidentes por quedas. Aproximadamente, um em cada três idosos cai uma vez ao ano ${ }^{(6)}$ e a chance de cair aumenta com a idade, principalmente a partir dos 80 anos, ${ }^{(7,8)}$ caracterizando-se como uma das principais causas de morbidade e mortalidade de idosos no mundo. ${ }^{(9)}$

A queda é definida como uma mudança inesperada de posição que leva o indivíduo a um nível inferior e resulta em risco de hospitalização ou até morte. ${ }^{(10)}$ Além do risco de hospitalizaçôes ou até mesmo de mortes, as quedas têm alto custo econômico e impacto significativo na utilização de serviços de saúde. ${ }^{(11)}$

Assim, investigar as gerontecnologias IoT no intuito de conhecer as opções tecnológicas disponíveis para impedir ou reduzir o quadro recorrente de quedas é relevante e necessário. Essas tecnologias podem promover inovação, expansão e aprimoramento do cuidado em saúde, melhoria da qualidade de vida dos idosos, suas famílias e cuidadores, permitindo, ainda, transformaçóes nas práticas dos profissionais de saúde, em especial de enfermeiros.

Nessa perspectiva, este estudo objetivou identificar na literatura as gerontecnologias IoT desenvolvidas para prevenção de acidentes por quedas em idosos.

\section{Métodos}

Trata-se de revisão integrativa, na qual foram adotadas as seguintes etapas: 1) Escolha da pergunta de pesquisa; 2) Definição dos critérios de inclusão e exclusão dos estudos; 3) Seleção da amostra; 4) Inclusão dos estudos selecionado; 5) Análise dos resultados, identificando diferenças e conflitos; 6) Discussão dos dados. ${ }^{(12)}$ Este estudo adotou as diretrizes da rede EQUATOR e seguiu as recomendaçōes da estratégia Preferred Reporting Items for Systematic Reviews and Meta-Analyses Preferred Reporting Items for Systematic Reviews and MetaAnalyses (PRISMA). ${ }^{(13)}$
A busca foi definida por meio da estratégia PIE ${ }^{(14)}$ (População, Intervenção, Efeito/Avaliação). Dessa forma, a populaçáo de interesse são idosos, a intervenção gerontecnologias IoT e a avalição é prevenção de acidentes por quedas, resultando na seguinte pergunta: "Quais as gerontecnologias IoT desenvolvidas para prevenção de acidentes por quedas em idosos disponíveis na literatura?".

Foram critérios de inclusão artigos sem restrição de período ou idioma com indivíduos de 60 anos ou mais, que abordem a utilização de gerontecnologia Internet das Coisas para prevençáo de quedas. Excluíram-se artigos duplicados. O período de coleta foi de janeiro a maio de 2020. A busca nas bases de dados foi realizada pelo acesso online e de forma independente por dois pesquisadores.

A pesquisa foi realizada nas seguintes bases de dados: Medical Literature Analysis and Retrieval System Online/National Library of Medicine (MEDLINE/ PubMed), Literatura Latino-Americana e do Caribe em Ciências da Saúde (LILACS), Cumulative Index to Nursing and Allied Health Literature (CINAHL), Scopus e Web of Science.

Para ampliar a busca, adotaram-se termos da linguagem natural, descritores não controlados e descritores controlados da terminologia em saúde dos Descritores em Ciências da Saúde (DeCS). O quadro 1 traz os termos e estratégia de busca nas bases.

Quadro 1. Componentes da estratégia PIE, termos e estratégia de busca e bases de dados

\begin{tabular}{|c|c|c|c|c|}
\hline \multirow[t]{2}{*}{ Componente } & \multicolumn{2}{|c|}{ Termos da busca } & \multirow{2}{*}{$\begin{array}{l}\text { Estratégia de } \\
\text { busca }\end{array}$} & \multirow[t]{2}{*}{ Bases } \\
\hline & $\begin{array}{c}\text { Descritores não } \\
\text { controlados }\end{array}$ & $\begin{array}{l}\text { Descritores } \\
\text { controlados }\end{array}$ & & \\
\hline P: População & $\begin{array}{l}\text { Elderly; Old; Elder; } \\
\text { Aging }\end{array}$ & Aged & \multirow{3}{*}{$\begin{array}{c}\text { (Gerontotechnology } \\
\text { OR Technology OR } \\
\text { Internet of Things) } \\
\text { AND (Elderly OR } \\
\text { Old OR Elder OR } \\
\text { Aged OR Aging) } \\
\text { AND Prevention AND } \\
\text { Accidents Fall; }\end{array}$} & \multirow{3}{*}{$\begin{array}{c}\text { MEDLINE/ } \\
\text { PubMed } \\
\text { LILACS } \\
\text { CINAHL } \\
\text { Web of } \\
\text { Science } \\
\text { SCOPUS }\end{array}$} \\
\hline I: Intervenção & Gerontotechnology & $\begin{array}{c}\text { Technology; } \\
\text { Internet of Things }\end{array}$ & & \\
\hline $\begin{array}{l}\text { E: Efeito/ } \\
\text { Avaliação }\end{array}$ & Prevention & Accidents Fall & & \\
\hline
\end{tabular}

Efetuou-se a leitura de título e resumo de todos os artigos rastreados; em seguida, realizou-se leitura minuciosa dos artigos selecionados e escolhidos aqueles para compor a amostra final, sendo elaborado um quadro síntese com as seguintes informaçóes para a análise das tecnologias: au- 
tor, local de utilização, ano de publicação, país de desenvolvimento, tipo de estudo, principais conclusôes, profissionais envolvidos, órgão de fomento, descrição, desfecho, especificação quanto a sensores, jogos sérios, exergames, dispositivos, realidade virtual, robôs, aplicativos e classificação em gerontecnologias Io $\mathrm{T}$ ou protótipos de gerontecnologia IoT.

Destaca-se que sensores são dispositivos sensíveis a alguma forma de interação com o ambiente que coletam informaçóes sobre uma medida física que precisa ser mensurada. São classificados em térmicos, de pressão, vestíveis, de velocidade, de posição, de aceleração, dentre outros. ${ }^{(15)}$

Jogos sérios: jogos virtuais com a finalidade educacional e de promoção da saúde, mas podem possuir os princípios gerais dos jogos, como divertimento e entreterimento. ${ }^{(16)}$

Exergames: estes necessitam da movimentação do corpo inteiro do indivíduo associada ao exercício físico com videogame. ${ }^{(17)}$

Dispositivos: objetos que estão relacionados a um conjunto de regras, palavras que se relacionam entre si e podem influenciar na conduta das pessoas. ${ }^{(18)}$

Realidade virtual: descrita como uma interface avançada do usuário, tendo como características a visualização e a movimentação em ambientes tridimensionais. ${ }^{(19)}$
Robô: máquina que realiza tarefas de repetição e que exijem precisão elevada. ${ }^{(20)}$

Aplicativos: conjunto de ferramentas delineado para executar ações específicas. ${ }^{(21)}$

Para a classificaçáo das gerontecnologias IoT e protótipos de gerontecnologia IoT, levou-se em consideração os seguintes elementos essencias para a funcionalidade de uma IoT: conectividade, sensoriamento e processamento. ${ }^{(4)}$ Portanto, estudos que apresentavam os três elementos foram considerados gerontecnologias IoT e estudos que apresentavam pelo menos um desses elementos, protótipos de gerontecnologias IoT. Protótipo pode ser caracterizado como uma versão preliminar de um novo produto desenvolvido. ${ }^{(22)}$

As informaçôes dos estudos foram sistematizadas, categorizadas e analisadas.

\section{Resultados}

Identificaram-se 1873 artigos e, após a leitura dos títulos e resumos, excluíram-se 1563 artigos, restando 310 para a etapa seguinte. Nessa etapa, realizou-se leitura completa, após a qual foram excluídos 287. Por fim, a amostra final desta revisão foi constituída por 23 estudos. A figura 1 apresenta o processo de seleçáo desta pesquisa de acordo com o fluxograma PRISMA.

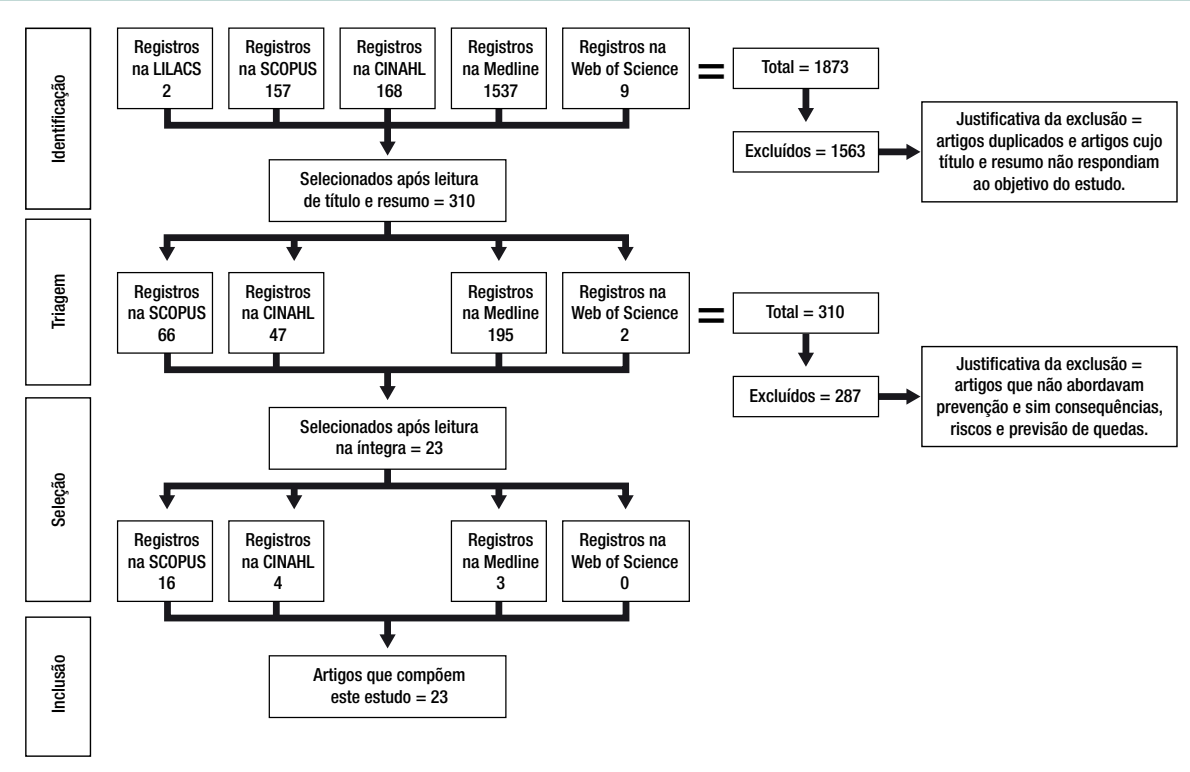

Figura 1. Fluxograma do Processo de Seleção dos Artigos 
As gerontecnologias IoT disponíveis na literatura para prevenção de acidentes por quedas foram publicadas entre 2008 e 2020, a maioria nos anos de 2018 e 2019, nos seguintes países: Austrália (3), ${ }^{(23-25)}$ Estados Unidos da América (3), ${ }^{(26-28)}$ Tailândia (2), ${ }^{(29,30)}$ Alemanha (2), ${ }^{(31,32)}$ Aústria (1), ${ }^{(33)}$ Suécia (1), ${ }^{(34)}$ Argélia (1), ${ }^{(35)}$ Canadá (1), ${ }^{(36)}$ Coreia do Sul (1), ${ }^{(37)}$ Finlândia (1), ${ }^{(38)}$ Japáo (1), ${ }^{(39)}$ Portugal (1), ${ }^{(40)}$ Reino Unido (1), ${ }^{(41)}$ Itália (1), ${ }^{(42)}$ Sérvia (1), ${ }^{(43)}$ Suíça $(1)^{(44)}$ e Taiwan (1). ${ }^{(45)}$

Das 23 gerontecnologias Iot identificadas, seis eram estudos descritivos, ${ }^{(26,27,32,36,38,40)}$ três estudos experimentais, ${ }^{(25,29,33)}$ um ensaio clínico randomizado, ${ }^{(42)}$ um quase-experimental ${ }^{(30)}$ e doze não relataram a metodologia utilizada. ${ }^{(23,24,28,31,34,35,37,39,41,43-45)}$

No que se refere à autoria dos estudos por categoria profissional, destacam-se ciência da computação (7), ${ }^{(23,26,28,31,32,34,35)}$ engenheiros (4), ${ }^{(24,27,38,43)}$ enfermeiros (1) ${ }^{(33)}$ e 12 artigos não informaram a formação dos autores. ${ }^{(25,29,30,36,37,39,40-42,44,45)}$

Quanto à instituição de fomento das gerontecnologias IoT, dez foram financiadas por instituições governamentais, ${ }^{(27,29,33,34,36,38,40,43-45)}$ duas por organização não governamental ${ }^{(23,26)}$ e duas por universidades. ${ }^{(30,41)}$ As demais não citaram. ${ }^{(25,28,31,32,35,37,39,42)}$

No tocante aos desfechos das gerontecnologias IoT, oito eram voltadas para mobilidade, ${ }^{(24,28,32,36,39,42-44)}$ seis para equilíbrio, ${ }^{(25,27,29,30,37,40)}$ quatro para os movimentos, ${ }^{(23,31,33,41)}$ três para detecção de objetos ${ }^{(26,35,45)}$ e uma para capacidade física. ${ }^{(38)}$ Todavia, um artigo não relatou para qual desfecho a gerontecnologia era destinada. ${ }^{(34)}$

Quanto ao local de desenvolvimento, cinco foram desenvolvidas em ambiente hospitalar ${ }^{(23,26,31-33)}$ e domiciliar, respectivamente ${ }^{(30,34,35,40,43)}$ duas em instituiçóes de atendimento ao idoso ${ }^{(42,44)}$ e onze não mencionaram. ${ }^{(24,25,27-29,36-39,41,45)}$

O quadro 2 traz as especificaçóes dos estudos referentes às gerontotecnologias do tipo IoT (oito) ${ }^{(24,26,31-35,44)}$ e protótipo de gerontecnologias (15), ${ }^{(23,25,27-30,36-43,45)}$ sendo sete sensores, ${ }^{(23,26,32,33,36-37,41)}$ cinco dispositivos, ${ }^{(25,39,42,44,45)}$ três jogos sérios ${ }^{(29,40,43)}$ e sistemas, ${ }^{(31,34,35)}$ dois robôs ${ }^{(27,30)}$ e um exergame, ${ }^{(38)}$ realidade virtual ${ }^{(24)}$ e aplicativo. ${ }^{(28)}$

\section{Discussão}

Nesta pesquisa, destaca-se o desenvolvimento de gerontecnologias IoT para prevenção de acidentes por quedas, publicadas, em sua maioria, nos últimos dois anos, ${ }^{(24,29-31,34-38)}$ ratificando o quão atual é o tema. A predominância de estudos, por engenheiros $^{(24,27,38,43)}$ e profissionais da ciência da computação, ${ }^{(23,26,28,31-35)}$ em países da Europa, ${ }^{(31-34,38,40-44)}$ América do Norte, ${ }^{(26-28,36)}$ Ásia ${ }^{(29,30,37,39,45)}$ e Oceania, ${ }^{(23-25)}$ comprova a seriedade do assunto em âmbito mundial e reforça a tendência de maior investimento tecnológico em países desenvolvidos.

Nesse sentido, a Austrália ${ }^{(23-25)}$ e Estados Unidos $^{(26-28)}$ apresentaram maiores produtividade de gerontecnologias IoT, o que pode ser entendido como uma resposta do país a esse agravo, já que as quedas são as principais causas externas de morte para idosos com mais de 85 anos nesse país. Buscar estratégias para prevenção de quedas são indispensáveis, visto que as açóes de prevenção podem proporcionar redução de $66 \%$ na sua incidência. ${ }^{(46)}$

Apesar desta revisão não apresentar estudos brasileiros, os dados sobre quedas em idosos são alarmantes. Em 2018, ocorreram 123.774 internaçóes hospitalares no país por quedas. ${ }^{(47)}$ A taxa de ocorrência de quedas varia de 10,7 a 59,3\% em idosos residentes na comunidade e de 32,5 a 66,7\% em residentes de Instituiçôes de Longa Permanência para Idosos. ${ }^{(48)}$

A partir dos resultados deste estudo, espera-se que profissionais de saúde, engenheiros e pesquisadores se motivem e busquem desenvolver pesquisas envolvendo gerontecnologias Io $\mathrm{T}$ destinadas à prevenção de quedas e divulguem seus resultados. As instituições de pesquisas, universidades, organizaçôes governamentais e não governamentais e iniciativa privada precisam investir no desenvolvimento de tecnologias voltadas à população idosa, uma vez que o aumento dessa população exige açôes, estratégias e mudanças nas políticas públicas, além de financiamento com editais e projetos específicos.

Destaca-se, ainda, que para incremento nessa área temática, faz-se necessário o estabelecimento de parcerias entre centros tecnológicos e profissio- 


\section{Quadro 2. Gerontecnologias loT e protótipos de Gerontotecnologia loT para prevenção de acidentes por quedas}

\begin{tabular}{|c|c|c|c|}
\hline \multicolumn{4}{|c|}{ Gerontecnologias loT } \\
\hline Ano/País/ Citação & Especificações & Descrição & Principais conclusões \\
\hline $\begin{array}{l}\text { Aidemark et al. } \\
\text { 2019/ Suécia }\end{array}$ & $\begin{array}{l}\text { Sistema- Gerenciamento de } \\
\text { quedas com aconselhamento, } \\
\text { sensores e aplicativo. }\end{array}$ & $\begin{array}{l}\text { Combinação de ações preventivas por meio de aconselhamento especializado e } \\
\text { exemplos de riscos, detecção de incidentes de queda com sensores que captam } \\
\text { movimentos corporais, detecção visual do movimento e posição do usuário, análise de } \\
\text { padrões de comportamento. }\end{array}$ & $\begin{array}{l}\text { Ambiente de aprendizagem no domicílio } \\
\text { orientado para loT para prevenção de quedas } \\
\text { que facilita a vida de forma independente e } \\
\text { autocompreensão aprimorada para facilitar a vida } \\
\text { cotidiana. Desenvolvido em ambiente domiciliar. }\end{array}$ \\
\hline $\begin{array}{l}\text { Aljahdali et } \\
\text { al.,2018/ Argélia(35) }\end{array}$ & $\begin{array}{l}\text { Sistema - Dispositivo walker } \\
\text { assistivo inteligente para } \\
\text { deficientes visuais e idosos. }\end{array}$ & $\begin{array}{l}\text { Dispositivo de hardware e software. Hardware composto por sensor, controlador } \\
\text { e saída. Software composto pelos aplicativos "Mapa" e "Encontre-me"Apps. As } \\
\text { informações recebidas dos sensores (posição, objetos e pessoas ao redor) são } \\
\text { enviadas a comandos para controlador e orientar o andador para atender às } \\
\text { necessidades dos idosos com segurança. }\end{array}$ & $\begin{array}{l}\text { Reconhece as posições perigosas que podem } \\
\text { causar a queda. Desenvolvido em ambiente } \\
\text { domiciliar. }\end{array}$ \\
\hline $\begin{array}{l}\text { Jähne-Raden et al., } \\
\text { 2019/ Alemanha }{ }^{(31)}\end{array}$ & Sistema INBED & $\begin{array}{l}0 \text { componente vestível é preso à parte superior da perna do idoso na metade superior } \\
\text { da coxa, ideal para detectar eventos de elevação por mudanças de ângulo das pernas } \\
\text { sem afetar o conforto. } \\
\text { Além do sensor, o sistema desenvolvido contém estações retransmissoras e uma estação } \\
\text { base de processamento de sinal central para criar uma rede de comunicação escalável. }\end{array}$ & $\begin{array}{l}\text { Sistema oferece apoio ao atendimento profissional } \\
\text { na redução de lesões de pacientes causadas por } \\
\text { quedas. Desenvolvido em ambiente hospitalar. }\end{array}$ \\
\hline $\begin{array}{l}\text { Kouris et al., 2015/ } \\
\text { Sérvia }^{(43)}\end{array}$ & $\begin{array}{l}\text { Jogo - KINOPTIM: Plataforma } \\
\text { de jogo que fornece o perfil } \\
\text { médico de cada indivíduo } \\
\text { para desenvolver um Sistema } \\
\text { de Apoio à Decisão Clínica } \\
\text { Personalizado (PCDSS). }\end{array}$ & $\begin{array}{l}\text { Durante a execução dos exercícios, os sensores vestíveis são colocados nos pulsos } \\
\text { e tornozelos para detecção da movimentação; em seguida, o módulo MBI incorpora } \\
\text { um Sistema de Apoio à Decisão (DSS) para coletar, organizar e analisar as relações } \\
\text { passivas, ativas e cooperativas dos dados coletados. O DSS utilizado na plataforma } \\
\text { KINOPTIM recebe como entrada o perfil médico de cada indivíduo para desenvolver } \\
\text { um Sistema Personalizado de Apoio à Decisão Clínica (PCDSS). }\end{array}$ & $\begin{array}{l}\text { Classifica níveis de risco de queda e gera } \\
\text { recomendações aos médicos. Desenvolvido em } \\
\text { ambiente domiciliar. }\end{array}$ \\
\hline $\begin{array}{l}\text { Torres et al., 2016/ } \\
\text { Austrália(23) }^{(23)}\end{array}$ & $\begin{array}{l}\text { Sensor - WISP sensor vestível } \\
\text { sem bateria com identificação } \\
\text { por radiofrequência (RFID). }\end{array}$ & $\begin{array}{l}\text { Sensor wearable sem bateria para uso sobre roupa anterior ao esterno, no qual } \\
\text { existem dados correspondendo aos seus movimentos e sua identificação sendo } \\
\text { coletados em tempo real via RFID. } \\
\text { Os dados são recebidos na fase de reconhecimento de saída da cama e da cadeira } \\
\text { para processamento e análise. Assim, pode ser emetido um alerta para cuidadores no } \\
\text { momento da saída da cama ou da cadeira. }\end{array}$ & $\begin{array}{l}\text { Determinar saídas de cama e cadeira em tempo real } \\
\text { é importante devido à falta de métodos na literatura } \\
\text { que usam sensores sem bateria para prevenção de } \\
\text { quedas. Desenvolvido em ambiente hospitalar. }\end{array}$ \\
\hline $\begin{array}{l}\text { Wolf et al., 2013/ } \\
\text { Alemanha }^{(32)}\end{array}$ & $\begin{array}{l}\text { Sensor - Alarme para saída } \\
\text { da cama. }\end{array}$ & $\begin{array}{l}\text { Sensores Shimmer disponíveis na beira do leito e equipados com Bluetooth. Protótipo } \\
\text { integra proteção de movimento amplo como precursor para saída da cama e proteção } \\
\text { de altas acelerações durante a queda. }\end{array}$ & $\begin{array}{l}\text { Detecta tentativas de sair da cama de maneira } \\
\text { confiável e gera poucos alarmes falsos. } \\
\text { Desenvolvido em ambiente hospitalar. } \\
\end{array}$ \\
\hline $\begin{array}{l}\text { Ranasinghe et } \\
\text { al.,2012/ EUA }{ }^{(26)}\end{array}$ & $\begin{array}{l}\text { Sensor - WISP: Plataforma de } \\
\text { identificação e detecção sem fio } \\
\text { por meio de um sensor vestível. }\end{array}$ & $\begin{array}{l}\text { Identificação e detecção sem fio da Plataforma WISPs (dispositivo vestível) para } \\
\text { monitoramento e identificação de atividades em tempo real: 1) Leitores RFID; 2) } \\
\text { Software de monitoramento. }\end{array}$ & $\begin{array}{l}\text { Grande potencial de fornecer uma intervenção } \\
\text { tecnológica para evitar quedas em hospitais. } \\
\text { Desenvolvido em ambiente hospitalar. }\end{array}$ \\
\hline $\begin{array}{l}\text { Hilbe et al., 2010/ } \\
\text { Aústriaa }^{(33)}\end{array}$ & $\begin{array}{l}\text { Sensor -BUCINATOR: alarme de } \\
\text { saída da cama. }\end{array}$ & $\begin{array}{l}\text { Trilhos sensíveis à pressão preenchidos com ar são montados na borda superior } \\
\text { da grade lateral da cama para o sistema de alarme de saída. Esses trilhos são } \\
\text { conectados com o sensor de pressão. Se um paciente com risco de queda pretende } \\
\text { sair da cama com a grade lateral na posição vertical ou abaixada, a pressão aumenta } \\
\text { no sistema e se um nível de limite for excedido, um sinal de alarme elétrico é gerado. } \\
\text { A equipe de enfermagem será informada sobre o risco da situação por meio do } \\
\text { sistema de chamada. }\end{array}$ & $\begin{array}{l}\text { Um sistema confiável de alarme de saída de cama } \\
\text { fornece confiança em relação à segurança de } \\
\text { seus parentes e sobrecarrega menos a família. } \\
\text { Desenvolvido em ambiente hospitalar. }\end{array}$ \\
\hline \multicolumn{4}{|c|}{ Protótipos de gerontecnologias loT } \\
\hline Ano/País/ Citação & Especificações & Descrição & Principais conclusões \\
\hline $\begin{array}{l}\text { Patton et al.,2008/ } \\
\text { EUA.(27) }\end{array}$ & $\begin{array}{l}\text { Robô - KineAssist para } \\
\text { treinamento de marcha e } \\
\text { equilíbrio. }\end{array}$ & $\begin{array}{l}\text { Fornece suporte parcial do peso corporal e controle postural; permite movimentos } \\
\text { do tronco e pelve; segue movimentos de caminhada sobre o solo nas direções para } \\
\text { frente, rotação e evasão; e impossibilita que } 0 \text { indivíduo perca o equilíbrio. }\end{array}$ & $\begin{array}{l}\text { Pode ajudar na prevenção das quedas, pois } \\
\text { melhora marcha e equilíbrio. Não cita o local de } \\
\text { desenvolvimento. }\end{array}$ \\
\hline $\begin{array}{l}\text { Merilampi } \\
\text { et al.,2019/ } \\
\text { Finlândia(38) }\end{array}$ & $\begin{array}{l}\text { Exergame - baseado em uma } \\
\text { cadeira inteligente. }\end{array}$ & $\begin{array}{l}\text { Desenvolvido para dispositivos Android via Bluetooth. Os movimentos do usuário } \\
\text { são medidos por nove sensores de pressão, localizados sob o assento da cadeira. } \\
\text { Os sensores enviam seus dados para o dispositivo Android. } 0 \text { exergame "cadeira } \\
\text { inteligente" utiliza três categorias de minijogos: salto de esqui, snowboarde } \\
\text { lançamento de bola. A interação com o ambiente do jogo exige que o usuário mova } \\
\text { seu corpo. } 0 \text { minigame de snowboard foi projetado para treinar controle postural na } \\
\text { posição sentada. } 0 \text { minijogo de salto de esqui requer maior habilidade funcional. } 0 \\
\text { jogo de lançamento de bola incorpora força e coordenação da extremidade superior. }\end{array}$ & $\begin{array}{l}\text { Facilita a entrega de ferramentas divertidas no } \\
\text { fornecimento de intervenções preventivas com } \\
\text { potencial para diminuir o risco de queda. Não cita o } \\
\text { local de desenvolvimento. }\end{array}$ \\
\hline $\begin{array}{l}\text { Prasertsakul } \\
\text { et al.,2018/ } \\
\text { Tailândia }{ }^{(29)}\end{array}$ & $\begin{array}{l}\text { Jogo - Treinamento de } \\
\text { equilíbrio de realidade virtual } \\
\text { de dupla tarefa (DTVRBT). }\end{array}$ & $\begin{array}{l}\text { Laptop e sensor Kinect. As informações 3D desse sensor permite que os usuários } \\
\text { interajam com o objeto no ambiente virtual (Unity3D). } 0 \text { jogo requer movimento do } \\
\text { braço nas direções vertical e horizontal. } 0 \text { jogador deve colocar } 0 \text { objeto na área } \\
\text { atribuída. }\end{array}$ & $\begin{array}{l}\text { Pode facilitar um melhor controle postural } \\
\text { e contribuir para a prevenção de quedas de } \\
\text { adultos saudáveis no futuro. Não cita o local de } \\
\text { desenvolvimento. }\end{array}$ \\
\hline $\begin{array}{l}\text { Verrusio et } \\
\text { al.,2017/ Itália(42) }\end{array}$ & $\begin{array}{l}\text { Dispositivo -Posturizador do } \\
\text { corpo humano (HBP) }\end{array}$ & $\begin{array}{l}\text { Dispositivo composto por órtese articulada, com quatro elementos que entram } \\
\text { em contato com diferentes zonas anatômicas, sendo capaz de adaptar-se às } \\
\text { características físicas de cada indivíduo. Melhora a precisão da resposta, caminhada e } \\
\text { postura, estimulando o córtex pré-frontal(PFC), fundamental no controle motor. }\end{array}$ & $\begin{array}{l}\text { Instrumento útil para a prevenção de quedas em } \\
\text { idosos, pois reduz fatores de risco em idosos com } \\
\text { condições crônicas. Desenvolvido em instituição } \\
\text { geriátrica. }\end{array}$ \\
\hline $\begin{array}{l}\text { Viriyavit et al.,2020/ } \\
\text { Reino Unido }\end{array}$ & $\begin{array}{l}\text { Sensor - Alarme para detecção } \\
\text { de saída da cama }\end{array}$ & $\begin{array}{l}\text { Painel de sensor equipado com dois tipos de sensores para detectar o peso aplicado } \\
\text { na cama, sendo instalado no lado esquerdo e no lado direito do painel. A combinação } \\
\text { dos dois pares de diferentes tipos de sensores é usada para detectar a posição de } \\
\text { cada lado do corpo na cama. }\end{array}$ & $\begin{array}{l}\text { Funciona com alta precisão na detecção de } \\
\text { posição e requer o menor número de sensores } \\
\text { com relação a outros existentes. Não cita o local de } \\
\text { desenvolvimento. }\end{array}$ \\
\hline $\begin{array}{l}\text { Raffe et al.,/ 2018/ } \\
\text { Austrália(24) }^{(24)}\end{array}$ & $\begin{array}{l}\text { Realidade virtual - Kinect } \\
+ \text { HTC Vive - Configuração } \\
\text { simples que combina } \\
\text { rastreamento esquelético e } \\
\text { realidade virtual. }\end{array}$ & $\begin{array}{l}\text { Kinect: rastrear o movimento corporal do jogador. HTC Vive: usado para a tela de } \\
\text { realidade virtual. }\end{array}$ & $\begin{array}{l}\text { Possui o potencial de aumentar a imersão de uma } \\
\text { experiência e prevenir possíveis quedas. Não cita } 0 \\
\text { local de desenvolvimento. }\end{array}$ \\
\hline
\end{tabular}




\begin{tabular}{|c|c|c|c|}
\hline \multicolumn{4}{|c|}{ Protótipos de gerontecnologias loT } \\
\hline Ano/País/ Citação & Especificações & Descrição & Principais conclusões \\
\hline $\begin{array}{l}\text { Tzung-Han et } \\
\text { al.,2017/ Taiwan }{ }^{(45)}\end{array}$ & $\begin{array}{l}\text { Dispositivo -Sapatos de } \\
\text { prevenção de quedas }\end{array}$ & $\begin{array}{l}\text { Laser de linha na lateral dos sapatos e câmera RGB na parte superior dos sapatos. } \\
\text { Sistema envia alerta de acordo com a largura e profundidade dos obstáculos } \\
\text { detectados. }\end{array}$ & $\begin{array}{l}\text { Adequado para ser instalado em dispositivos } \\
\text { portáteis e custos gerais dos produtos são } \\
\text { aceitáveis } \\
\text { Não cita o local de desenvolvimento. }\end{array}$ \\
\hline $\begin{array}{l}\text { Di P et al., 2016/ } \\
\text { Japão }\end{array}$ & $\begin{array}{l}\text { Dispositivo - Bengala } \\
\text { inteligente }\end{array}$ & $\begin{array}{l}\text { Robô constituído por base móvel unidirecional, bastão de alumínio, minicomputador, } \\
\text { sistema de sensores e um telêmetro a laser (LRF), utilizado para auxiliar idosos no } \\
\text { treinamento da caminhada. }\end{array}$ & $\begin{array}{l}\text { Comporta-se para impedir que o usuário esteja } \\
\text { em posição que gere quedas. Não cita o local de } \\
\text { desenvolvimento. }\end{array}$ \\
\hline $\begin{array}{l}\text { Vieira et al.,2015/ } \\
\text { Portugal(40) }\end{array}$ & $\begin{array}{l}\text { Jogo - baseado no teste Berg } \\
\text { Balance Scale (BBS) }\end{array}$ & $\begin{array}{l}\text { Associaçao de jogo + Sensor de movimento baseado no BBS que consiste em } \\
\text { exercícios de tarefas contidianas. E os sensores são utilizados para detecção do } \\
\text { movimento, distinguindo corretamente entre as atividades da vida diária, como sentar } \\
\text { ou caminhar, de uma duração real de queda. }\end{array}$ & $\begin{array}{l}\text { Melhora a autonomia e a segurança, } \\
\text { especificamente para aqueles em risco de queda. } \\
\text { Desenvolvido em ambiente domiciliar. }\end{array}$ \\
\hline $\begin{array}{l}\text { Maneeprom } \\
\text { et al.,2019/ } \\
\text { Tailândia(30) }^{30}\end{array}$ & Robô & $\begin{array}{l}\text { Tela de toque de } 8 \text { polegadas fixada na cabeça de um robô Dinsow Mini versão } \\
\text { M2070, em que os idosos assistem a quatro vídeos sobre prevenção de quedas e } \\
\text { escolha de dispositivos de auxílio à caminhada, dois vídeos sobre calçado adequado, } \\
\text { dois vídeos sobre exercícios e } 28 \text { mensagens de voz sobre prevenção de quedas e } \\
\text { lembrente de exercícios diários. }\end{array}$ & $\begin{array}{l}\text { Aumenta o conhecimento sobre prevenção de } \\
\text { quedas, promover exercícios e melhorar o equilíbrio } \\
\text { entre idosos fisicamente ativos e em risco de queda } \\
\text { Desenvolvido em ambiente domiciliar. }\end{array}$ \\
\hline $\begin{array}{l}\text { Qiu et al.,2018/ } \\
\text { Coreia do Sul(37) }\end{array}$ & $\begin{array}{l}\text { Sensores -Conjunto de } \\
\text { sensores inerciais vestíveis em } \\
\text { miniatura }\end{array}$ & $\begin{array}{l}\text { Sensores inerciais são colocados sobre a parte inferior das costas, coxas e pernas } \\
\text { e realizada bateria de testes para avaliação multifatorial de risco de queda para } \\
\text { identificar o maior número de fatores de risco apresentados. }\end{array}$ & $\begin{array}{l}\text { Sistemas baseados em sensores inerciais vestíveis } \\
\text { mostram grandes promessas para a avaliação de } \\
\text { prevenção de queda em idosos. Não cita o local de } \\
\text { desenvolvimento. }\end{array}$ \\
\hline $\begin{array}{l}\text { Wu et al., 2018/ } \\
\text { Canadá( }{ }^{(6)}\end{array}$ & $\begin{array}{l}\text { Sensor - Programa de } \\
\text { avaliação de risco de queda } \\
\text { baseada em sensor vestivel } \\
\text { (SFRA). }\end{array}$ & $\begin{array}{l}\text { SFRA consiste em duas peças centrais: dispositivo vestível para coleta e } \\
\text { armazenamento de dados e um dispositivo móvel para exibição de informações de } \\
\text { risco de queda. }\end{array}$ & $\begin{array}{l}\text { Informações podem motivar os idosos a adotar } \\
\text { intervenções de prevenção de quedas. Não cita o } \\
\text { local de desenvolvimento. }\end{array}$ \\
\hline $\begin{array}{l}\text { Moufawad et } \\
\text { al.,2016/ Suíç̧(44) }\end{array}$ & $\begin{array}{l}\text { Dispositivo-Sapatos } \\
\text { instrumentados }\end{array}$ & $\begin{array}{l}0 \text { sistema consiste na unidade de detecção de inércia e registro de dados Physilog } \\
\text { e a palmilha de pressão plantar. Sua arquitetura é orientada para coletar dados do } \\
\text { calçado em tempo real e realizar a classificação e caracterização da atividade online } \\
\text { (por exemplo, análise da marcha dos períodos de locomoção e análise da transição } \\
\text { posturall). Os dados de todos os sensores (calcanhar medial e lateral, arco lateral, } 1^{\circ} \text { / } \\
3^{\circ} / 5^{\circ} \text { metatarsais, hálux e os dedos restantes) são armazenados em um cartão de } \\
\text { memória para evitar perdas de dados. }\end{array}$ & $\begin{array}{l}\text { Avalia a mobilidade e fornece feedback rápido } \\
\text { e personalizado para idosos. Desenvolvido em } \\
\text { instituição geriátrica. }\end{array}$ \\
\hline $\begin{array}{l}\text { Goulding et } \\
\text { al.,2015 EUA' }\end{array}$ & Aplicativo - Sistema Sparrow. & $\begin{array}{l}\text { Aplicativo móvel para Android conectado a sensores e acoplado a uma cadeira de } \\
\text { rodas que emite sinais de movimentação alertando sobre o risco de queda. }\end{array}$ & $\begin{array}{l}\text { Eficiente para emitir alerta reais sobre a } \\
\text { movimentação do usuário durante seu uso. Não cita } \\
\text { o local de desenvolvimento. }\end{array}$ \\
\hline $\begin{array}{l}\text { Qiu et al.,2013/ } \\
\text { Austrália(25) }\end{array}$ & $\begin{array}{l}\text { Dispositivo - Palmilhas } \\
\text { texturizadas }\end{array}$ & Palmilhas inseridas em calçados padronizados para reduzir a oscilação postural. & $\begin{array}{l}\text { Melhora a estabilidade e reduz a oscilação postural. } \\
\text { Não cita o local de desenvolvimento. }\end{array}$ \\
\hline
\end{tabular}

nais de saúde, ressaltando a participação de enfermeiros nesse processo. Nesta revisão, a participação de enfermeiros foi escassa. No entanto, sabe-se que sua expertise no cuidado poderá agregar ao aparato tecnológico o desenvolvimento de tecnologias mais sensíveis às necessidades dos idosos. ${ }^{(49)}$

Observou-se, neste estudo, quanto às gerontecnologias IoT produzidas em diferentes ambientes, que cerca de $30 \%$ das quedas estão relacionadas ao ambiente físico. ${ }^{(50)}$ As gerontecnologias produzidas que se voltaram à detecçáo precoce de anormalidades no movimento dos idosos e ao declínio de suas habilidades biomecânicas são possíveis para identificar comprometimentos da locomoção que podem levar a quedas. Nesse sentido, gerontecnologias IoT específicas voltadas às limitaçôes funcionais já existentes podem possibilitar melhora contínua da capacidade funcional, reduzindo as consequências da falta de equilíbrio no idoso. ${ }^{(51)}$

As gerontecnologias IoT atuam de forma mais eficaz no acompanhamento e monitoramento de idosos. ${ }^{(52)}$ As aplicaçôes clínicas das gerontecnologias IoT na saúde de idosos incluem monitorização, diagnóstico, previsão de agravos e tratamento. Por exemplo, por meio da gerotecnologia IoT, o sistema ou um dispositivo pode detectar sinais de alerta de risco referente aos idosos e enviar um sinal ou mensagem para cuidadores e profissionais da saúde. Dessa forma, pode acompanhar remotamente e em tempo real seu estado de saúde. ${ }^{(53)}$

Destaca-se que para a construção de soluçóes IoT são necessárias outras tecnologias, ${ }^{(54)}$ necessitando, assim, de mão de obra especializada, investimentos e parcerias no intuito de produzir tecnologias de acordo com as demandas necessárias.

A arquitetura de soluçóes IoT deve abranger elementos complexos, como conectividade, sensoriamento e processamento, o que se torna um desafio para o seu desenvolvimento e implementação. Dada a heterogeneidade dos objetos, a dificuldade na transmissão de dados em tempo real, análise instantânea de dados realizada no pré-processamento, inteligên- 
cia local em sistemas embarcados de baixa potência, interação entre usuários no local, interfaces complexas, portabilidade e vestibilidade, ${ }^{(32)}$ muitos produtos são desenvolvidos abrangendo um ou mais elementos citados, configurando-se, assim, como protótipos.

Destaca-se a grande quantidade de gerontecnologias IoT com a utilização de sensores ${ }^{(23,26,32,33,36,37,41)}$ e dispositivos. ${ }^{(25,39,42,44,45)} \mathrm{O}$ uso de sistemas de acionamento de alertas baseados em sensores é uma abordagem promissora que pode informar à equipe de enfermagem, familiares e cuidadores quando um paciente tenta sair da cama. ${ }^{(55)}$ Sensores eletrônicos de detecção para prevenção de quedas de pacientes relacionados à cama estão se tornando cada vez mais comuns e são projetados para detectar pacientes que saem da cama sem assistência, sendo capazes de evitar situaçóes perigosas. ${ }^{(56)}$ Os sensores dessa natureza são de baixo custo e de fácil aquisição, pois podem ser utilizados sensores de presença comuns, disponíveis em lojas de eletrônicos, por exemplo.

Nesta revisão, os dispositivos foram projetados com o intuito de melhorar a estabilidade postural e consequentemente prevenir quedas. Os dispositivos também podem fornecer uma alternativa eficaz e de baixo custo para a redução da oscilação postural e avaliação da marcha em pessoas idosas. ${ }^{(57)}$

As gerontotecnologias IoT que utilizaram robôs, ${ }^{(27,30)}$ jogos sérios, ${ }^{(29,40,43)}$ realidade virtual ${ }^{(24)} \mathrm{e}$ exergames $^{(38)}$ apareceram em menor número, talvez por seu desenvolvimento envolver maior tempo e custos mais elevados, contudo possuem diversas possibilidades de utilização na atenção e cuidado à pessoa idosa. Os jogos, por exemplo, oferecem uma maneira atraente e divertida de aprender, além de auxilar no entendimento que a tecnologia pode melhorar a autonomia e a segurança, avaliar o risco e ajudar a prevenir possíveis quedas, contribuindo, dessa forma, para o envelhecimento saudável. ${ }^{(15)}$

Assim, as gerontecnologias IoT podem ser utilizadas como ferramentas importantes para auxiliar na prevenção de quedas e no fortalecimento da capacidade funcional. Os resultados da revisão reforçam a necessidade de práticas interprofissionais para melhor atender às necessidades da população idosa.

Salienta-se a originalidade do estudo por se tratar de uma temática atual e pouco explorada no cenário brasileiro. $\mathrm{O}$ método adotado possibilita a seleção de diferentes tipos de estudos, o que por um lado enriquece os achados, todavia restringe os detalhamentos dos mesmos, pois não há exigência de critérios quanto a qualidade e força das evidências, impossibilitando, assim, informaçóes mais aprofundadas quanto ao desenvolvimento da gerontecnologia Io T, limitando a oportunidade de replicação por outros pesquisadores.

\section{Conclusão}

Evidenciou-se que o período de 2018 e 2019 apresentou maior número de publicaçóes, destacando-se a produção na Austrália. Ressalta-se a predominância de estudos descritivos, engenheiros e profissionais da ciência da computação como os que mais produziram. Para prevenção de quedas, salienta-se as gerontecnologias IoT voltadas a mobilidade e equilíbrio. Encontraram-se gerontecnologias IoT que abordavam sistemas, sensores, dispositivos, jogos sérios, exergames, robôs, realidade virtual e aplicativos. Por ser um tema ainda em desenvolvimento, fazem-se necessárias pesquisas futuras que busquem analisar a eficácia das gerontecnologias IoT para prevenção de quedas, estudos que fomentem discussóes sobre avaliação de custos e aplicabilidade na população. Além da necessidade de estabelecimento de parcerias entre centros tecnológicos e profissionais de saúde.

\section{Referências}

1. De La Torre F, Morales D, Quiroz CP. Gerontecnología: rapid review y tendencias mundiales. Rev Mex Ing Biomed. 2015;36(3):171-9.

2. Piau $A$, Campo $E$, Rumeau $P$, Vellas $B$, Nourhashémi $F$. Aging society and gerontechnology: a solution for an independent living? J Nutr Health Aging. 2014;18(1):97-112. Review.

3. Ilha S, Santos SS, Backes DS, Barros EJ, Pelzer MT, Gautério-Abreu DP. Gerontechnologies used by families/caregivers of elderly people with Alzheimers: contribution to complex care. Texto Contexto Enferm. 2018; 27(4):e5210017.

4. Al-Fuqaha A, Guizani M, Mohammadi M, Aledhari M, Ayyash M. Internet of things: a survey on enabling technologies, protocols, and applications. IEEE Commun Surv Tutor. 2015;17(4):2347-76.

5. Yang $\mathrm{H}$, Lee W, Lee H. loT smart home adoption: the importance of proper level automation. J Sensors. 2018;6464036:1-11. 
6. Almeida LM, Meucci RD, Dumith SC. Prevalence of falls in elderly people: a population based study. Rev Assoc Med Bras (1992). 2019;65(11):1397-403.

7. Gullich I, Cordova DD. Queda em idosos: estudo de base populacional. Rev Soc Bras Clin Med. 2017;15(4):230-4.

8. Alshammari SA, Alhassan AM, Aldawsari MA, Bazuhair FO, Alotaibi FK, Aldakhil AA, et al. Falls among elderly and its relation with their health problems and surrounding environmental factors in Riyadh. J Family Community Med. 2018;25(1):29-34.

9. James SL, Lucchesi LR, Bisignano C, Castle CD, Dingels ZV, Fox JT, et al. The global burden of falls: global, regional and national estimates of morbidity and mortality from the Global Burden of Disease Study 2017. Inj Prev. 2020;26(Supp 1):i3-i11.

10. Nascimento JS, Tavares DM. Prevalência e fatores associados a quedas em idosos. Texto Contexto Enferm. 2016;25(2):1-9.

11. Pirrie M, Saini G, Angeles R, Marzanek F, Parascandalo J, Agarwal G. Risk of falls and fear of falling in older adults residing in public housing in Ontario, Canada: findings from a multisite observational study. BMC Geriatr. 2020;20(1):11.

12. Botelho LL, Cunha CC, Macedo M. 0 método da revisão integrativa nos estudos organizacionais. GeS. 2011;5(11):121-36.

13. Galvão TF, Pansani TS, Harrad D. Principais itens para relatar revisões sistemáticas e meta-análises: a recomendação PRISMA. Epidemiol Serv Saúde. 2015;24(2):335-42.

14. Easy as PIE. Nursing. 1999;29(4):25.

15. Harshavardhan B, Reddy D, Joseph C. Sensor types and its applications. Intern J Pharm Technol. 2016;8(4):20172-80.

16. Money AG, Atwal A, Boyce E, Gaber S, Windeatt S, Alexandrou K. Falls Sensei: a serious 3D exploration game to enable the detection of extrinsic home fall hazards for older adults. BMC Med Inform Decis Mak. 2019;19(1):85.

17. Medeiros P, Capistrano R, Zequinão MA, Silva AS, Beltrame TS, Cardoso FL. Exergames as a tool for the acquisition and development of motor skills and abilities: a systematic review. Rev Paul Pediatr. 2017;35(4):464-71.

18. Dodier N, Barbot J. A força dos dispositivos. Soc Estado. 2017;32(2):487-518.

19. Schiavoni JE. Realidade virtual e lógica do espaço. Galaxia (São Paulo). 2018;39:165-76.

20. Siqueira-Batista R, Souza CR, Maia PM, Siqueira SL. Robotic surgery: bioethical aspects. ABCD Arq Bras Cir Dig . 2016;29(4):287-90.

21. Banos 0, Villalonga C, Garcia R, Saez A, Damas M, Holgado-Terriza JA, et al. Design, implementation and validation of a novel open framework for agile development of mobile health applications. Biomed Eng Online. 2015;14(Suppl 2):S6.

22. Canuto da Silva G, Kaminski PC. Selection of virtual and physical prototypes in the product development process. Int $\mathrm{J}$ Adv Manuf Technol. 2016;84:1513-30.

23. Torres RL, Visvanathan R, Hoskins $S$, van den Hengel A, Ranasinghe DC. Effectiveness of a Batteryless and Wireless Wearable Sensor System for Identifying Bed and Chair Exits in Healthy Older People. Sensors (Basel). 2016;16(4):546.

24. Raffe WL, Garcia JA. Combining skeletal tracking and virtual reality for game-based fall prevention training for the elderly. 2018 IEEE 6th International Conference on Serious Games and Applications for Health (SeGAH). Vienna: IEEE; 2018. p. 1-7. doi: 10.1109/ SeGAH.2018.8401371.
25. Qiu F, Cole MH, Davids KW, Hennig EM, Silburn PA, Netscher H, et al. Effects of textured insoles on balance in people with Parkinson's disease. PLoS One. 2013;8(12):e83309.

26. Ranasinghe DC, Shinmoto Torres RL, Sample AP, Smith JR, Hill K, Visvanathan R. Towards falls prevention: a wearable wireless and battery-less sensing and automatic identification tag for real time monitoring of human movements. Annu Int Conf IEEE Eng Med Biol Soc. 2012;2012:6402-5.

27. Patton J, Brown DA, Peshkin M, Santos-Munné JJ, Makhlin A, Lewis E, et al. KineAssist: design and development of a robotic overground gait and balance therapy device. Top Stroke Rehabil. 2008;15(2):131-9.

28. Goulding T, Deligiannidis L. Sparrow: a smart device for fall prevention. 2015 International Conference on Computational Science and Computational Intelligence (CSCI). Las Vegas (USA): IEEE; 2015. p. 797-9. doi: 10.1109/CSCI.2015.82.

29. Prasertsakul T, Kaimuk P, Chinjenpradit W, Limroongreungrat W, Charoensuk W. The effect of virtual reality-based balance training on motor learning and postural control in healthy adults: a randomized preliminary study. Biomed Eng Online. 2018;17(1):124.

30. Maneeprom N, Taneepanichskul S, Panza A, Suputtitada A. Effectiveness of robotics fall prevention program among elderly in senior housings, Bangkok, Thailand: a quasi-experimental study. Clin Interv Aging. 2019;14:335-46. Review.

31. Jähne-Raden N, Kulau U, Marschollek M, Wolf KH. INBED: a highly specialized system for bed-exit-detection and fall prevention on a geriatric ward. Sensors (Basel). 2019;19(5):1017.

32. Wolf KH, Hetzer K, Zu Schwabedissen HM, Wiese B, Marschollek M. Development and pilot study of a bed-exit alarm based on a body-worn accelerometer. Z Gerontol Geriatr. 2013;46(8):727-33.

33. Hilbe J, Schulc E, Linder B, Them C. Development and alarm threshold evaluation of a side rail integrated sensor technology for the prevention of falls. Int J Med Inform. 2010;79(3):173-80.

34. Aidemark J, Askenäs L. Fall Prevention as personal learning and changing behaviors: systems and technologies. Procedia Computer Science. 2019;164:498-507.

35. Aljahdali M, Abokhamees R, Bensenouci A, Brahimi T, Bensenouci M. IoT based assistive walker device for frail \&visually impaired people. 2018 15th Learning and Technology Conference (L\&T). Saudi Arabia: IEEE; 2018. p. 171-7. doi: 10.1109/LT.2018.8368503.

36. Wu AY, Munteanu C. Understanding older users' acceptance of wearable interfaces for sensor-based fall risk assessment. Conference Human Factors Computing Systems. 2018;119:1-13.

37. Qiu H, Rehman RZ, Yu X, Xiong S. Application of wearable inertial sensors and a new test battery for distinguishing retrospective fallers from non-fallers among community-dwelling older people. Sci Rep. 2018;8:16349.

38. Merilampi S, Mulholland K, Ihanakangas V, Ojala J, Valo P, Virkki J. A smart chair physiotherapy exergame for fall prevention - user experience study. 2019 IEEE 7th International Conference on Serious Games and Applications for Health (SeGAH). Japan: IEEE; 2019. p. 1-5. doi: 10.1109/SeGAH.2019.8882482.

39. Di P, Hasegawa Y, Nakagawa S, Sekiyama K, Fukuda T, Huang J, et al. Fall Detection and Prevention Control Using Walking-Aid Cane Robot. IEEE/ASME Transactions Mechatronics. 2016;21(2):625-37.

40. Vieira B, Pereira L, Freitas R, Terroso M, Simoes R. A gamified application for assessment of balance and fall prevention. 2015 10th Iberian Conference on Information Systems and Technologies (CISTI). Portugal: IEEE; 2015. p. 1-6. Doi: 10.1109/CISTI.2015.7170473. 
41. Viriyavit W, Sornlertlamvanich V. Bed position classification by a neural network and bayesian network using noninvasive sensors for fall prevention. J Sensors. 2020;5689860:1-14.

42. Verrusio W, Gianturco V, Cacciafesta M, Marigliano V, Troisi G, Ripani $M$. Fall prevention in the young old using an exoskeleton human body posturizer: a randomized controlled trial. Aging Clin Exp Res. 2017;29(2):207-14.

43. Kouris I, Tsirbas C, Tagaris T, Vellidou E, Vartholomeos P, Rizou S, et al. KINOPTIM: The medical business intelligence module for fall prevention of the elderly. 2015 IEEE 15th International Conference on Bioinformatics and Bioengineering (BIBE). Serbia: IEEE; 2015. p. 1-4. doi: 10.1109/BIBE.2015.7367637.

44. Moufawad El Achkar C, Lenoble-Hoskovec C, Major K, ParaschivIonescu $A$, Büla $C$, et al. Instrumented shoes for real-time activity monitoring applications. Stud Health Technol Inform. 2016;225:663-7.

45. Tzung-Han L, Chi-Yun Y, Wen-Pin S. Fall prevention shoes using camera-based line-laser obstacle detection system. J Healthc Eng. 2017;8264071:1-11.

46. Mackenzie L, Clifford A. Perceptions of the elderly in Ireland and Australia on the use of technology to combat fall prevention. Ageing Soc. 2020;40(2):369-88.

47. Brasil. Ministério da Saúde. Datasus. Informações de Saúde (TABNET). Brasília (DF): Ministério da Saúde; 2019 [citado 2020 Jul 20]. Disponível em: http://www2.datasus.gov.br/DATASUS/index.php?area=0205

48. Leitão SM, Oliveira SC, Rolim LR, Carvalho RP, Coelho Filho JM, Peixoto Junior AA. Epidemiology of falls in older adults in Brazil: an integrative literature review. Geriatr Gerontol Aging. 2018;12(3):172-9.
49. Olympio PC, Alvim NA. Board games: gerotechnology in nursing care practice. Rev Bras Enferm. 2018;71(2):818-26.

50. Mahmoodabad SS, Zareipour M, Askarishahi M, Beigomi A. Effect of the living environment on falls among the elderly in Urmia. Open Access Maced J Med Sci. 2018;6(11):2233-8.

51. Khanuja K, Joki J, Bachmann G, Cuccurullo S. Gait and balance in the aging population: fall prevention using innovation and technology. Maturitas. 2018;110:51-6. Review.

52. Borelli E, Paolini G, Antoniazzi F, Barbiroli M, Benassi F, Chesani F, et al. Habitat: an loT solution for independent elderly. Sensors. 2019;19(5):1258

53. Pasluosta CF, Gassner H, Winkler J, Klucken J, Eskofier BM. An emerging age in Parkinson's disease management: wearable technologies and the internet of things. IEEE J Biomed Health Inform. 2015;19:1873-81.

54. da Costa CA, Pasluosta CF, Eskofier B, da Silva DB, da Rosa Righi R. Internet of health things: toward intelligent vital signs monitoring in hospital wards. Artif Intell Med. 2018;89:61-9. Review.

55. Alam MF, Katsikas S, Beltramello 0, Hadjiefthymiades S. Augmented and virtual reality based monitoring and safety system: a prototype IoT platform. J Netw Comput Appl. 2017;89(1):109-19.

56. Mileski M, Brooks M, Topinka JB, Hamilton G, Land C, Mitchell T, et al. Alarming and/or alerting device effectiveness in reducing falls in long-term care (LTC) facilities? A systematic review. Healthcare (Basel). 2019;7(1):51.

57. Poier PP, Godke F, Foggiatto JA, Ulbricht L. Development and evaluation of low cost walker with trunk support for the elderly. Rev Esc Enferm USP. 2017;51:e03252. 